\title{
Proposed terminology for the classification and parameters for the quantification of variability in ionosphere morphology
}

\author{
Peter A. Bradley $\left(^{1}\right)$ and Ljiljana R. Cander $\left(^{2}\right)$ \\ (1) Pandora, Farnham Common, Slough, Berks, U.K. \\ ( $\left.{ }^{2}\right)$ Rutherford Appleton Laboratory, Chilton, Didcot, Oxon, U.K.
}

\begin{abstract}
Much uncertainty currently exists in the use by different workers of the term 'variability' in describing the results of statistical analyses applied to ionospheric measurement data sets and in their relationships with various existing or new ionospheric models. Often it is not clear whether data for different time periods or different geographical areas, and if so which, are being used to formulate results. Terms are presented in the Annex which it is suggested should be used, at least once in every publication addressing this topic, to describe unambiguously what is talked about. The background to the proposed terminology is discussed. Options for variability parameters are also addressed.
\end{abstract}

Key words ionosphere-ionospheric terminologyspace and time variations

\section{Introduction}

Parameters characterising ionospheric phenomena are subject both to periodic and irregular temporal variations. Ionospheric characteristics from which the height profiles of electron density may be reconstituted for a given location, and vertical total electron content, which is the height-integrated density within unit cross section, experience systematic changes with time-of-day, season and epoch of the solar cycle together with so-called 'random' fluctuations about these regular states. There are also small-scale variations with geographical position

Mailing address: Dr. Ljiljana R. Cander, Rutherford Appleton Laboratory, Radio Communications Research Unit, Chilton, Oxon OX11 0QX, U.K.; e-mail: 1.cander@rl.ac.uk as well as major repeatable geographical dependencies.

For aeronomy investigations and for applications involving the planning and performance assessment of telecommunication systems there is need to quantify both the regular and irregular changes that take place. In most cases such information comes from past measurements which are incomplete in space and time, and where there is need to use matched empirical expressions to provide complete coverage. It is usual to assume that future conditions may be estimated by extrapolation from the past with long-term predictions involving estimates of monthly median conditions based on some solar index. Variations about these monthly median figures are quantified statistically. On the other hand, there also exist requirements for short-term forecasts over various time intervals of up to a few hours from the present where a number of current studies are addressing approaches involving update of recent past 'instantaneous' measurements 
in terms of some near real-time space weather or geomagnetic index monitor.

So, as well as modelling median states and generating forecasting algorithms, various different types of temporal and spatial variability specification are needed. These include the separate applications of quantifying the match of median models to past or future measurement data sets from the same or different locations, the statistical characterisation of the day-to-day changes at a given hour and for a given place about these median models, the degree of match of forecasting algorithms to the data sets used in their formulation, a measure of the 'goodness' of a particular forecasting algorithm taken from the match to other data sets, and for aeronomy studies measures of variations in time and space over various continuous periods and distances. Unfortunately most relevant models and measurement data sets embody a mixture of the above described temporal and spatial changes, so that in order to obtain the above sorts of variability information various approximations have to be applied, with considerable opportunities for error or confusion. This paper aims to present a standardised classification of the different variability types, to be used in future studies to minimise the above problems that have already been identified in certain past analyses.

Whilst any varying data set can be specified in terms of its associated amplitude probability distribution, such information is too unwieldy and unnecessary for most ionospheric applications. Reference distribution parameters are offered quantifying variability magnitude and distribution skew, from which if needed the full distribution or specific percentile points may be given approximately.

\section{Requirements}

Ionosphere electron densities vary significantly, both geographically at a given time, and at a given location at separate times. The changes that take place are also different at different heights because of the varying relative production, loss and movement influences, and so this means they are not the same for the individual ionospheric characteristics. How then can profile variability be specified?

Many efforts are directed at generating electron-density height profile models. Most are monthly median models, in which for a particular place the separate relevant ionospheric characteristics are modelled as a function of latitude, longitude, time-of-day, season and epoch of the solar cycle (as defined in terms of an appropriate solar-cycle index). All geographical locations are covered, and profiles are reconstituted in terms of these characteristics values using best-available empirical algorithms. The basic input data to the models come from past vertical-sounding or topside measurements, from theoretical considerations involving mean reaction rates and estimates of ionising fluxes, or from some combination of these various data sources.

A different case is where models are wanted for individual occasions, such as a particular day and time. In these instances it is usual to generate values of the separate ionospheric characteristics for the occasion of interest, and then to formulate the corresponding height profile on the assumption that all the various mean algorithms still apply, an exercise that at best has to be approximate. When concerned with past epochs, individual characteristics values, so-called 'instantaneous values' may come from maps produced for example through the application of geographical smoothing applied to discrete sounding results (Samardjiev et al., 1993).

Ionospheric characteristics instantaneousvalue maps, including where needed maps of TEC, and the associated electron-density height profiles may apply for specific past epochs, or they may incorporate a forecast element, and be for a future time. Likewise, monthly median characteristics maps and corresponding profile models may be used to 'predict' conditions, past or future, for different epochs than those associated with their formulation.

From the foregoing it is seen that a number of different sources of variability exist depending on the time periods and geographical locations being considered. It is usual to categorise changes as 'periodic' (or predictable) when the causes can be identified and included in the models, and so-called 'random' when the dependencies are 
not known, or are believed to arise from the combination of a number of individual factors that cannot be separately quantified.

Within the frameworks discussed above for a given ionospheric characteristic, we can consider a number of specific scenarios, for each of which we suppose the quoted variability combination is determined:

i) Past monthly median values and a monthly median model based on measured data for the same epochs and same geographical areas gives a measure of model accuracy in matching input data, useful in quantifying model uncertainties.

ii) Past monthly median values and a monthly median model based on measured data for different epochs, or different geographical areas gives a measure of model consistency for other situations, useful in establishing the extent of model applicability for possible improvement.

iii) Long-term future prediction and a monthly median model - predicted figures are taken to be monthly median values and the variability combination gives a measure of prediction accuracy.

iv) Continuous measured data sequence with values quoted at regular time or regular spatial intervals - gives a measure of data variability averaged over whatever period or area is considered.

v) Past measured instantaneous values and a monthly median model for matched or disparate epochs and geographical areas - gives a measure of model applicability to specifying instantaneous values.

vi) Extrapolated forecast from recent past measured instantaneous values - gives a measure of forecasting accuracy.

Further comments can be offered as follows: When considering regular daily-hourly ionosonde measurements, data for successive hours are influenced by the systematic diurnal changes and this should be borne in mind if quoting variability statistics from such information. Data sequences over shorter periods may provide useful information, for example concerning the extent of ionospheric disturbances, gravity and planetary waves (Forbes et $a l ., 2000)$. In such cases the sampling duration must always be quoted. Measured values for different days of a given month at a particular hour are often treated as an indication of day-to-day variability, yet such data sets also embody the influences of within-the-hour variability because the hourly ionosonde readings are essentially 'snapshots' of ionospheric state over the minute or less that a sounding takes. Within-the-hour variability is usually, but not always, less than dayto-day variability. This limitation should always be mentioned when discussing any such results. Storm and quiet-day values may be included within the variability statistics or treated as base references from which other day or other location values depart. TEC data sets taken from orbiting satellites include additional factors contributing to the variability, depending on how frequently measurements are recorded and the analyses procedures that are introduced to give these.

When considering the variability of electrondensity distributions, height profiles can be examined to yield electron density statistics at a series of different heights, or height distributions established for a series of different densities. The profiles may come either from direct measurements (Ezquer et al., 2002; Mosert et al., 2002), or be obtained by reconstitution from the individual ionospheric characteristics values. Amalgamation of the variability combinations for the different ionospheric characteristics does not seem feasible because of lack of knowledge of the associated correlation between different characteristics.

From the foregoing, the following cases are suggested as particularly appropriate for study:

- Day-to-day variability of $f o F 2, M(3000) F 2$, $f_{O F} 1$ and $f_{O E} E$ for selected ionosonde stations and hours, taken from measured hourly vertical soundings and without attempted correction for within-the-hour variability (e.g., Kouris et al., 2000a). These statistics are of potential value to the generation of improved propagation prediction procedures, where probability of ionospheric support is sought. Correlation between the different characteristics, if any, needs also to be established. Definitive figures for the most appropriate time and geographical groupings could usefully complement existing models.

- Day-to-day variability of TEC for selected GPS measuring stations and hours, provided by 
fOF2(MHz) 5 JUN 20011200 UT

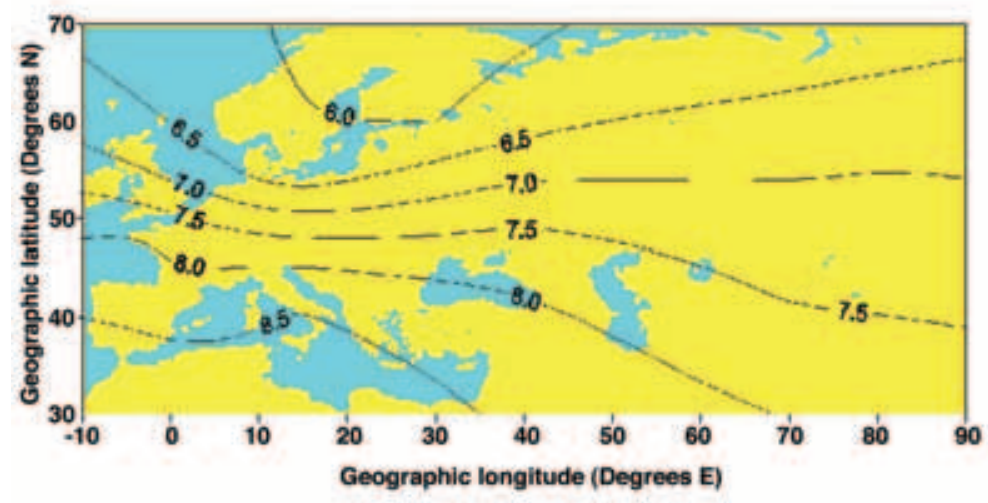

MUF(3000)F2/MHz) 5 JUN 20011200 UT

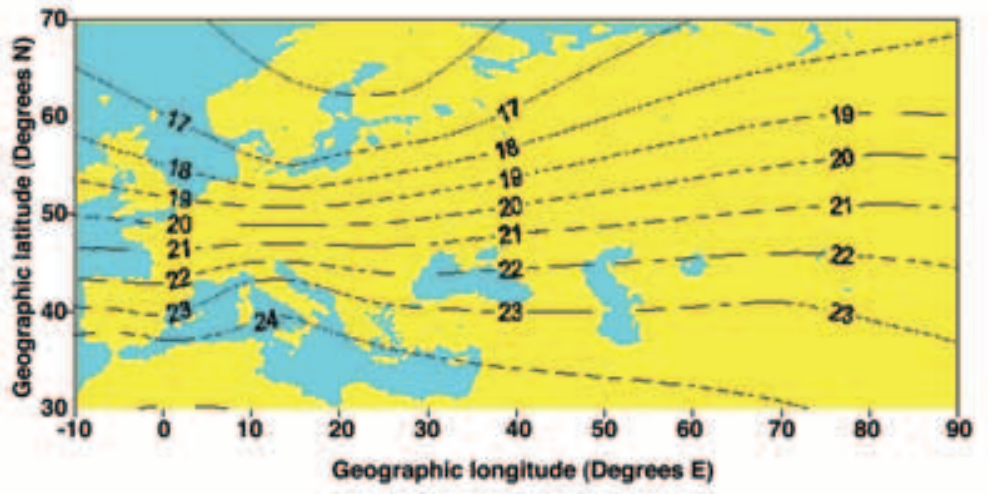

$\operatorname{TEC}\left(10^{\prime \prime} \mathrm{m}^{-2}\right) 5$ JUN 20011200 UT

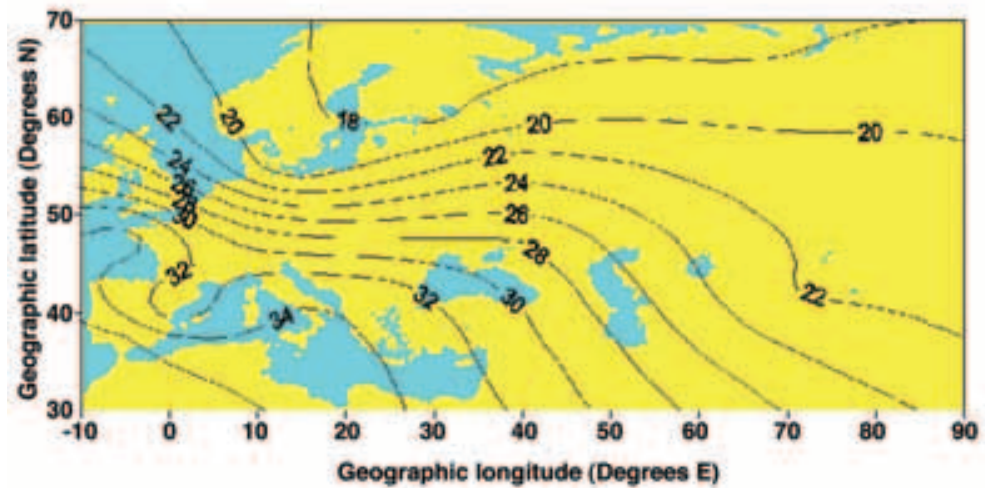

Fig. 1. Maps derived from $24 \mathrm{~h}$ ahead forecast of $f o F 2, \operatorname{MUF}(3000) F 2$ and TEC for 5 June 2001 at 1200 UT (http://ionosphere.rcru.rl.ac.uk). 

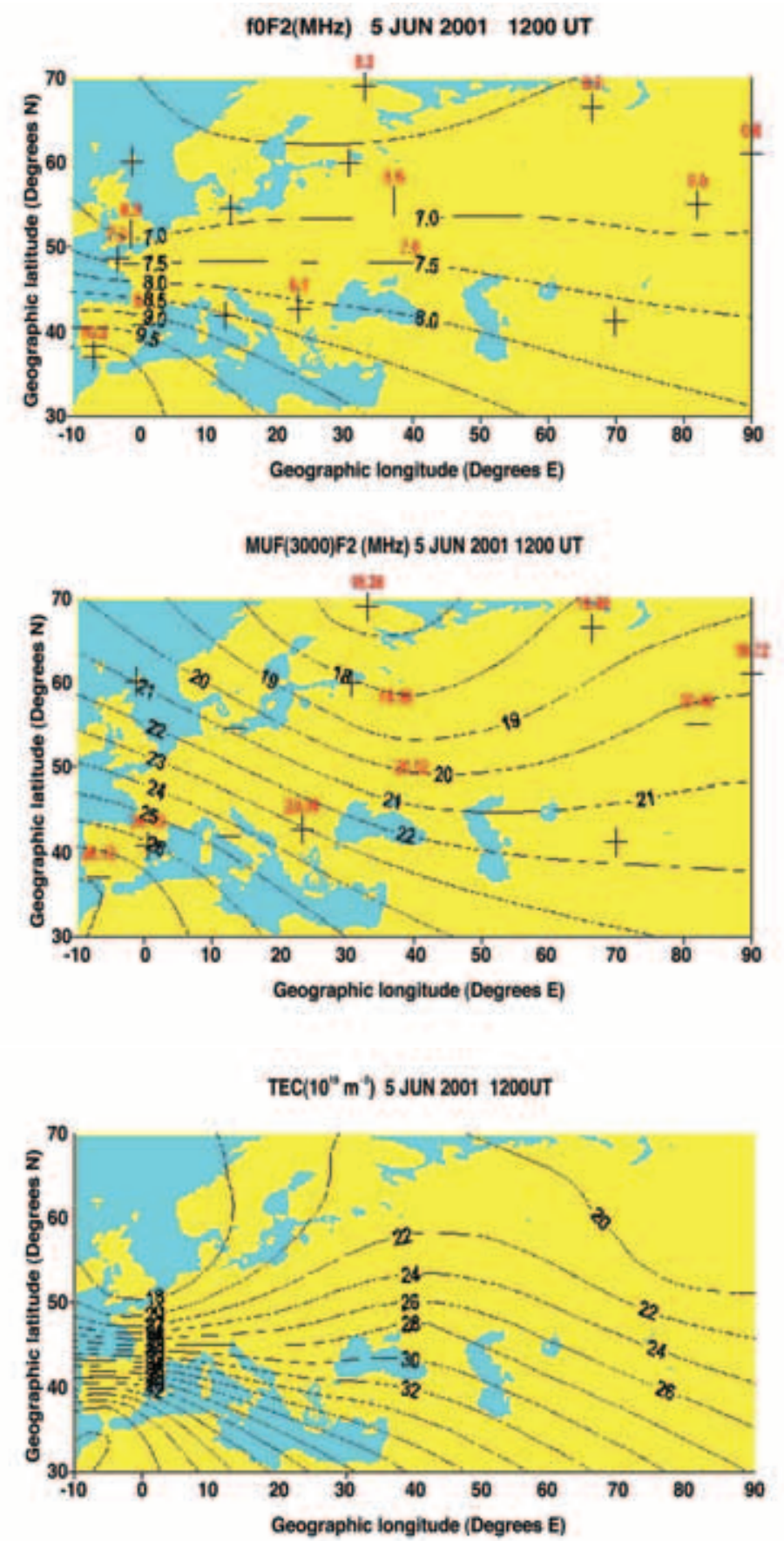

Fig. 2. Maps derived from measured values of $f o F 2, \operatorname{MUF}(3000) F 2$ and TEC for 5 June 2001 at 1200 UT (http://ionosphere.rcru.rl.ac.uk). 
the International GPS Service for Geodynamics (IGS). These statistics are also of potential value to the design and operation of improved Earthspace communication systems. At present often TEC is determined by height profile integration. It may well be that ultimately a global TEC model will be created based on direct measurements, perhaps along the lines of those already produced for Europe (Leitinger and Feichter, 2000; Cander, 2001).

- Variability between past monthly median values derived from measurements of the above ionospheric characteristics and values given from model specifications for different epochs and stations. These statistics can be interpreted as providing an indication of model accuracy.

- Variability among measured data sequences of the above ionospheric characteristics for continuous time periods of a few hours (Kouris et al., 2000b). These statistics provide a measure of short-term variations over whatever period or area is considered and are useful confidence figures to augment mapped characteristics values.

- Variability between past measured instantaneous mapped values of the above ionospheric characteristics and models for matched or disparate epochs and geographical areas (figs. 1 and 2). These statistics provide a measure of model applicability to specifying instantaneous values.

- Variability of electron density on different days of a given month at a particular hour for a series of different $F$-region heights. This information should be particularly valuable in establishing those heights at which variability is a minimum, and so aid especially in formulating improved algorithms to specify $F$-region thickness.

\section{Variability parameters}

Periodic changes are not usually considered in determining variability factors but it is the random variations that are quantified. A given data set consisting of 'randomly' varying scalar quantities $X$ is only fully specified by its complete amplitude-probability distribution. However, such information is unwieldy and not necessary for most applications. The set may though usefully be characterised by its mean value (or median value $X_{m}$ ), together with some measure of the spread about that central figure. One variability parameter is the overall standard deviation $\sigma$ related to the Root-Mean-Square Error (RMSE). A more refined specification includes a measure of distribution skew, with upper and lower distribution standard deviations $\sigma_{u}$ and $\sigma_{d}$ (or decile deviations $D_{u}$ and $D_{l}$ ) being separately quoted.

Ways of re-constituting the full distribution from median and standard or decile deviations assuming a particular distribution law exist and are adequate for many purposes. In the past, pairs of half-normal distributions have been adopted for the upper and lower sections, and use of the chi-squared distribution has been advocated (Zacharisen and Crow, 1970). However, the numerical complexity this involves is considered unjustifiable. Simple empirical formulae

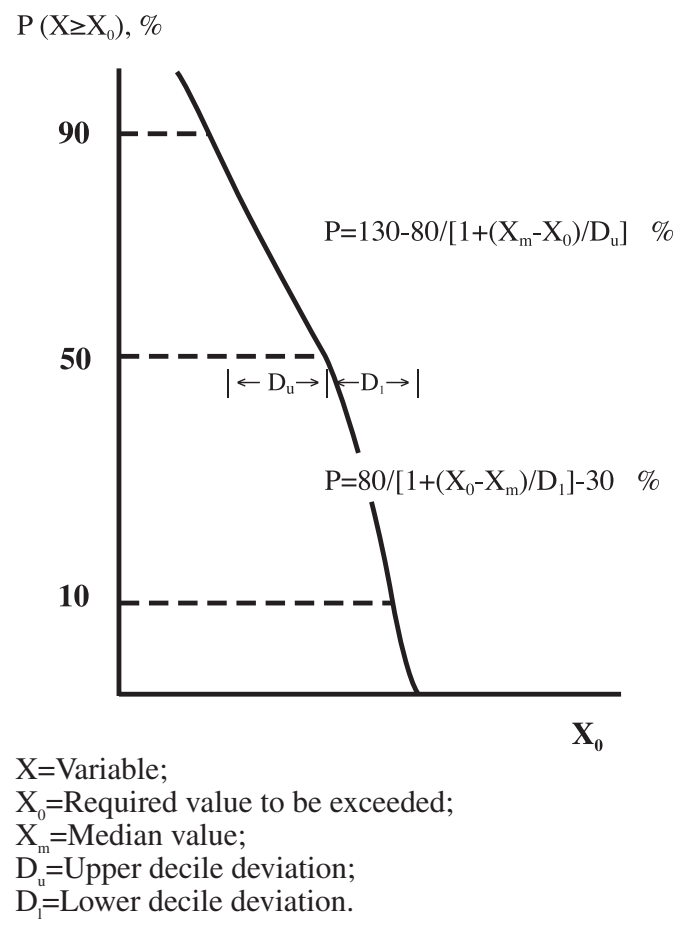

Fig. 3. Idealised cumulative-amplitude probability distribution fitted to median and decile deviation values, from Bradleyy and Bredford (1976). 
approximating to a pair of normal distributions developed by Bradley and Bedford (1976) and based entirely on use of $X_{m}, D_{u}$ and $D_{l}$ have been adopted by the ITU-R (2000) in a number of cases where day-to-day variability of ionospheric propagation parameters needs to be specified (fig. 3). Often it is useful to express the spread parameter $(\rho$ or $D)$ as a fraction of the median or mean value.

Indicated variability figures are obviously influenced by the length of the data series, particularly if long-period systematic changes are present and by sampling rates if a continuous sequence is approximated by a succession of 'spot values', as say in standard hourly verticalincidence ionospheric soundings, or regularinterval satellite probe measurements. Which quantities to quote will depend on the application, but in many cases it is suggested use of the $\left(X_{m}, D_{u}\right.$ and $\left.D_{l}\right)$ variability combination will provide all that is needed; sometimes the decile range $D_{u}-D_{l}$ is a useful quantity; other requirements call for specification of extreme 'worst case' maxima and minima of a particular data set.

\section{ANNEX}

\section{Proposed variability terminology}

Monthly median model accuracy variability quantification of match between monthly median model and measurement data for the same epochs and same geographical areas.

Monthly median model applicability variability - quantification of match between monthly median model and measurement data for different past epochs and the same geographical area, or for different geographical areas and the same epochs.

Long-term prediction accuracy variability quantification of match between monthly median model and measurement data for future epochs and the same or different geographical areas.

Short-term forecasting accuracy variability quantification of match between forecast values from recent past measured instantaneous values for the same or different geographical areas.

Continuous time-period variability - quantification of variations in measurement data sequence for the same location, with values quoted at regular time intervals.

Spatial variability - quantification of variations in measurement data sequences for the same epoch, with values quoted at regular location separations.

Composite measurement variability - quantification of combined variations in measurement data sequences for different locations and times.

Note: In all cases a full specification of the measurement data used should be provided.

\section{REFERENCES}

BRADlEy, P.A. and C. BEDFORD (1976): Prediction of HF circuit availability, Electron. Lett., 12, 32-33.

CANDER, LJ. R. (2001): TEC specification over Europe: A procedure, in IEE Eleventh International Conference on Antennas and Propagation, Manchester, IEE Publication, No. 480, 802-805.

EZQUER, R.G., M. MOSERT, S.M. RADICELLA and C.A. JADUR (2002): Study of the electron density variability at fixed heights over San Juan and Tucuman, Adv. Space Res., 29 (6), 993-997.

Forbes, J.M., S.E. PALO and X. ZHANG (2000): Variability of the ionosphere, J. Atmos. Sol.-Terr. Phys., 62, 685693.

ITU-R (2000): HF propagation prediction method, Recommendation ITU-R P. 533, International Telecommunication Union, Geneva, Switzerland.

Kouris, S. S, D.N. Fotiadis and R. HANBABA (2000a): On the day-to-day variations of the MUF over Europe, Phys. Chem. Earth, C, 25 (4), 319-325.

Kouris, S.S., B. Zolesi, D.N. Fotiadis and C. Bianchi (2000b): On the variability within-the-hour and from hour-to-hour of the $F$-region characteristics above Rome, Phys. Chem. Earth, C, 25 (4), 347-351.

Leitinger, R. and E. Feichter (2000): COSTTEC - the electron content monthly median map adopted by COST251, Phys. Chem. Earth, C, 25 (4), 311-314.

Mosert, M., S.M. RAdicella, D. BuRESOVA, R.G. EZQUer and C.A. JADUR (2002): Study of the variations of the electron density at $170 \mathrm{~km}$, Adv. Space Res., 29 (6), 937941.

SAMARDJIEV, T., P.A. BRADlEy, LJ.R. CANDER and M.I. DiCK (1993): Ionospheric mapping by computer contouring techniques, Electron. Lett., 29 (20), 1794-1975.

ZACHARISEN, D. H. and E.L. CROW (1970): Fitting distributions of telecommunication variables with chisquare distributions, Radio Sci., 5 (11), 1307-1315. 